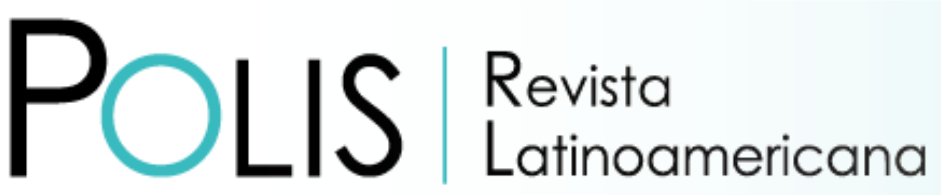

V20 | N58 | 2021

DOI: $10.32735 /$ S0718-6568/2021-N58-1583

\title{
Las largas trayectorias de cuidado remunerado de las familias peruanas en Córdoba, Argentina
}

\author{
María José Magliano \\ Centro de Investigaciones y Estudios sobre Cultura y Sociedad (CONICET y UNC), Córdoba, \\ Argentina. Email: mariajosemagliano@gmail.com \\ Denise Zenklusen \\ Centro de Investigación y Transferencia Rafaela (CONICET y UNRaf), Rafaela, Argentina. \\ Email: denisezenklusen@gmail.com
}

Recibido: 12.04.2020 | Aceptado: 24.07.2020

\begin{abstract}
Resumen: Este artículo se propone analizar las trayectorias de mujeres peruanas en el sector de los cuidados remunerados y sus continuidades generacionales. En base a un trabajo cualitativo longitudinal con mujeres migrantes de origen peruano que viven en barrios urbanos relegados de la ciudad de Córdoba (Argentina), y retomando los aportes teóricos de la perspectiva interseccional, indaga en la articulación entre migraciones, generaciones y trabajos de cuidado remunerados. El artículo muestra, primero, la centralidad de esos trabajos en las experiencias migratorias de mujeres peruanas, pioneras en muchos casos de la migración a Córdoba; segundo, el modo en que esos trabajos son heredados por sus hijas y las dificultades que estas jóvenes enfrentan para acceder y moverse hacia otros rubros del mercado de trabajo. Como conclusión, reflexiona sobre los alcances generacionales de la segregación laboral en las sociedades contemporáneas y la reproducción de desigualdades sociales asociadas a los trabajos de cuidado.
\end{abstract}

Palabras clave: Migraciones; género; generaciones; trabajos de cuidado remunerados.

\section{The Long-Paid Caregiving Trajectories of Peruvian families in Cordoba, Ar- gentina}

\begin{abstract}
The aim of this paper is to analyze the trajectories of Peruvian women in paid care work and their generational continuities. Based on a qualitative longitudinal work with migrant women of Peruvian origin who reside in relegated urban neighborhoods of the city of Córdoba (Argentina), and considering the contributions of intersectional perspective, it problematizes the articulation between migrations, generations and paid care work. The paper shows first, the role of care jobs in the migration experiences of Peruvian women, who were in many cases pioneers of the migration to Cordoba; second the way in which these jobs are inherited by their daughters and the difficulties these young women face in accessing and moving to other areas of the labor market. To conclude, it reflects on the generational impact on the labor segregation in contemporary societies and the reproduction of social inequalities related to care work.
\end{abstract}

Keywords: Migration; gender; generation; paid care work. 


\section{As longas trajetórias do cuidado remunerado das famílias peruanas em Córdoba, Argentina}

Resumo: Este artigo tem como objetivo analisar as trajetórias das mulheres peruanas no setor de cuidados remunerados e suas continuidades geracionais. Baseado em um trabalho longitudinal qualitativo com mulheres migrantes de origem peruana que vivem em bairros urbanos relegados da cidade de Córdoba (Argentina), e retomando às contribuições teóricas da perspectiva interseccional, reflete sobre a articulação entre migrações, gerações e cuidados remunerados. O artigo mostra, primeiro, a centralidade desses trabalhos nas experiências migratórias das mulheres peruanas, pioneiras em muitos casos de migração para Córdoba; segundo, a maneira como esses empregos são herdados pelas filhas dessas mulheres e as dificuldades que essas jovens enfrentam para ter acesso a outras áreas do mercado de trabalho. Concluindo, o artigo reflete sobre os alcances geracionais da segregação do trabalho nas sociedades contemporâneas e a reprodução das desigualdades sociais associadas aos trabalhos de cuidado.

Palavras-chave: Migrações; gênero; gerações; trabalhos de cuidado remunerados.

Como citar este artículo:

Magliano, M y Zenklusen, D. (2021). Las largas trayectorias de cuidado remunerado de las familias peruanas en Córdoba, Argentina. Polis Revista Latinoamericana, 20 (58), 160-176. doi: http://dx.doi.org/10.32735/S0718-6568/2021-N58-1583

\section{Introducción}

La migración pervana a la Argentina en general y a Córdoba en particular ha mostrado en los últimos tres decenios un gran dinamismo. Asociada desde sus inicios a motivaciones de carácter laboral (Falcón y Bologna, 2013; Rosas y Gil Araujo, 2019) y a un contexto de crisis económica y violencia política transitada por Perú en la década del noventa del siglo pasado (Cerrutti, 2005; Falcón y Bologna, 2013; Rosas, 2010); la migración peruana se distinguió por una fuerte feminización -que en el transcurso de los dos primeros decenios del siglo XXI se transformó en una migración de tipo familiar- en el marco de una demanda persistente de mujeres para trabajar en el sector de los cuidados. Esa demanda se mantiene hasta el presente, siendo los trabajos de cuidado la inserción laboral principal para las mujeres peruanas en Córdoba. En este sentido, en este artículo nos proponemos analizar sus trayectorias en el sector de los cuidados remunerados en Córdoba, así como sus continuidades generacionales.

En particular, intentaremos mostrar, en primer lugar, la centralidad de los trabajos de cuidado remunerados en las experiencias migratorias de mujeres peruanas, pioneras en muchos casos de la migración a Córdoba; en segundo lugar, el modo en que esos trabajos son heredados por sus hijas y las dificultades que estas jóvenes enfrentan para acceder y moverse hacia otros rubros del mercado de trabajo en el contexto de destino. Finalizaremos con una reflexión sobre los alcances de la segregación laboral presente en las sociedades contemporáneas a partir de los activos procesos de racialización, etnización y generización que recaen sobre determinadas poblaciones. 
En el campo de estudios sobre migraciones y género, la categoría cuidado ha recibido una importante atención en tanto un amplio conjunto de las mujeres migrantes a nivel global se dedica a los trabajos que componen ese sector laboral. Estas investigaciones repusieron la relevancia de los trabajos de cuidado (remunerados y no remunerados) en la organización y sostenimiento del proyecto migratorio. Dentro de las migraciones Sur-Norte, en particular, se analizó el rol de las mujeres migrantes en la provisión de los cuidados y la generación de cadenas transnacionales de cuidado (Catarino y Oso, 2000; Gutiérrez-Rodríguez, 2010; Herrera, 2016; Hochschild, 2000; Parreñas, 2001, Parella, 2007, entre muchos otros) como parte de los efectos del envejecimiento de la población en los países de destino, el aumento de la tasa de actividad de mujeres y la insuficiente oferta de estructuras públicas de cuidado (Pérez Orozco, 2007; Borgeaud-Garciandía, 2017). Estos estudios, a su vez, pusieron el acento en las crisis sociales y económicas atravesadas por los países de origen de la población migrante, en especial en el contexto de auge neoliberal, que convirtieron a la migración en una estrategia de subsistencia individual y familiar.

A diferencia de lo sucedido en estas latitudes, el peso de las cadenas globales de cuidado en las migraciones intrarregionales sudamericanas, como el caso abordado en este artículo, adquiere otros sentidos, en especial porque los procesos de reagrupación familiar suelen ser más rápidos. El hecho de que la familia en su conjunto se encuentre residiendo en Córdoba es lo que nos permitió reconstruir las continuidades generacionales en torno a los trabajos de cuidado remunerados. Trabajos que, como veremos más adelante, surgen como opción laboral a partir de la migración, convirtiéndose en la inserción principal para las mujeres que arriban desde Bolivia, Paraguay y Perú'. Asimismo, el foco puesto en estas largas trayectorias de cuidado entre las mujeres de las familias pervanas permite mostrar que la organización de la reproducción social descansa en el género a la vez que se encuentra atravesada por líneas de fracturas de clase, raza, sexualidad y nación (Arruza, Bhattacharya y Fraser, 2019, p. 41). Esto implica reconocer que, si bien los trabajos de cuidado son un terreno fundamentalmente femenino, las mujeres lo ocupan de forma diferenciada en tanto existe una jerarquía con base en la clase y origen social, el color de la piel, la nacionalidad (Dutra y Matos, 2016).

La categoría cuidados, aun cuando es difícil de asir representa una fuerza indubitable, en tanto se corresponde con las dimensiones, sentidos y complejidades de la realidad social (Borgeaud-Garciandía, 2018, p. 17). Tal como advierte Borgeaud-Garciandía (2017), las teorías del cuidado -que surgen en Estados Unidos en la década del ochenta a partir de los estudios de Carol Gilligan- se caracterizan en la actualidad por una diversidad de enfoques (Borgeaud-Garciandía, 2017). Entre esos enfoques, en este artículo nos apoyamos en aquellos que reflexionan sobre el cuidado como un trabajo. En esta línea, y retomando los aportes de Molinier (2018, p. 187), concebimos al cuidado no simplemente como una disposición o una ética, sino ante todo como un trabajo. En particular, un trabajo que

1 Para ampliar sobre los múltiples vínculos y cruces entre migraciones y cuidados en Argentina, véase Borgeaud-Garciandía (2017), Courtis y Pacecca (2010), Magliano, Perissinotti y Zenklusen (2016), Rodríguez Enríquez y Sanchís (2011). 
comprende todas aquellas actividades orientadas al sostenimiento cotidiano de la vida humana (Martín Palomo, 2016; Pérez Orozco, 2014; Vega y Gutiérrez-Rodríguez, 2014). Contemplando específicamente la dimensión remunerada de los trabajos de cuidado, nuestras interlocutoras peruanas se concentran en tres actividades principales: el trabajo doméstico remunerado, orientado fundamentalmente a la limpieza; la asistencia de niños y ancianos remunerado en casas particulares; y el cuidado comunitario, también remunerado, dirigido al sostenimiento de los espacios barriales destinados al cuidado de los niños (guarderías) y a la alimentación infantil (comedores y merenderos).

La tesis de este artículo propone que las largas trayectorias de cuidado remunerado de mujeres migrantes peruanas, expresadas en las escasas opciones de movilidad laboral y en sus continuidades generacionales, son una muestra de los procesos de segregación laboral a través de los cuales se legitima la concentración de las poblaciones migrantes en determinados sectores del mercado de trabajo y en las jerarquías laborales más precarizadas (Baeza, 2013; Magliano, 2017; Trpin y Pizarro, 2017). La segregación laboral, siguiendo este argumento, expresa una de las consecuencias centrales de los activos y cotidianos procesos de generización, etnización y racialización de la fuerza laboral².

Con miras a profundizar en las trayectorias laborales migrantes en el sector de los cuidados remunerados, el artículo se organiza en torno a tres apartados. En el primero, describiremos el anclaje teórico y metodológico del estudio. El segundo y el tercero se meten de lleno en el análisis de las trayectorias de las mujeres peruanas en los trabajos de cuidado remunerado en clave de larga duración. Por un lado, nos enfocaremos en la reconstrucción de las experiencias y trayectorias laborales en el sector de los cuidados de las mujeres peruanas adultas, quienes en muchas ocasiones encabezaron la migración hacia Córdoba. En especial, nos interesa indagar en las formas de acceso a esos trabajos y sus expectativas propias y familiares en relación con las posibilidades laborales en el contexto de destino. Por el otro, haremos foco en las "hijas de la migración" (Pedone, 2014) -esto es, en las jóvenes pervanas que llegaron a Córdoba en el marco de proyectos migratorios familiares- y en los vínculos que van estableciendo desde muy temprana edad con los trabajos de cuidado remunerados.

\section{Apuntes teóricos y metodológicos}

Las reflexiones que organizan este artículo se apoyan en los aportes teóricos de la perspectiva interseccional. Esta perspectiva, que surge al calor de las luchas de los feminismos

\footnotetext{
2 En estas páginas, la categoría género expresa las relaciones y jerarquías de poder basadas en las diferencias socialmente construidas entre lo femenino y lo masculino. La categoría raza, así como los procesos de racialización, hallan su fundamento en formas de dominación ancladas en la historia colonial y en los propios recorridos de las historias nacionales, las cuales se hallan inscritas en la relatividad de los cuerpos, que es histórica y debe ser contextualizada (Segato, 2007, p. 23). La etnicidad y los procesos de etnización, finalmente, se basan en jerarquías que se configuran en torno a aspectos culturales. La raza, al igual que ciertas categorías étnicas, es una construcción simbólica que se utiliza en ciertas circunstancias socio-políticas como criterio de definición y delimitación de grupos humanos (Stolcke, 2000, p. 8). Para profundizar sobre los efectos de la segregación laboral en las poblaciones migrantes, véase Magliano y Mallimaci (2018).
} 
negros en los Estados Unidos en las décadas del sesenta y setenta del siglo pasado, ofrece herramientas para analizar las posiciones desiguales que ocupan las personas en diferentes espacios de lo social, siendo el mercado de trabajo un lugar privilegiado para reconstruir esas posiciones ${ }^{3}$. La interseccionalidad pone en el centro de la discusión las bases materiales de la desigualdad -históricamente situadas- a la vez que destaca el carácter relacional de las posiciones y clasificaciones sociales, complejizando los enfoques que pretenden reducir las experiencias de los y las sujetos/as a través del lente de una sola categoría (Magliano, 2015).

La mirada interseccional en el campo de los estudios sobre el trabajo pone en evidencia que las posibilidades de que las personas obtengan un empleo dependen no sólo de su capital humano sino también de su género, origen étnico-nacional, estatus legal, edad, sexualidad, ubicación y otros criterios no económicos (Castles, 2013). En esta misma dirección es que Mezzadra y Neilson (2016, p.165) enfatizan la imposibilidad de considerar a los trabajadores como "sujetos neutrales que existen independientemente de las relaciones de poder ligadas al género, a la etnia, a la raza que se encuentran inscritas en sus cuerpos". Así, la intersección de esas formas de clasificación social condiciona y predispone a los/as sujetos/as migrantes, sus formas migratorias y sus oportunidades de inserción en el mercado de trabajo (Magliano y Mallimaci, 2018).

Esto se plasma en las trayectorias de mujeres migrantes en los trabajos de cuidado remunerados. La extendida inserción de la población femenina migrante en estos trabajos, como bien sugiere Borgeaud-Garciandía (2017, p. 21), "saca a relucir con particular intensidad el tema de la división social del trabajo y sus inequidades que se basan en las divisiones sociales de género, clase y raza asignadas". Estas divisiones sociales promueven "relaciones de subordinación y jerarquización en sus relaciones laborales" (Fulladosa-Leal, 2013, p. 25), al mismo tiempo que condenan a quienes realizan estos trabajos a la invisibilización, informalidad y desprotección. El cuidado resulta, en este marco, un trabajo cuyo análisis histórico y presente revela la profundidad de las desigualdades que caracterizan su distribución social, la cual recae en mujeres, aunque no en todas, y en mayor parte en aquellas que son pobres y/o migrantes y carecen de voz pública legítima (Borgeaud-Garciandía, 2018, p. 1415). Precisamente, la interseccionalidad ofrece herramientas teóricas para reflexionar críticamente sobre las asimetrías laborales que se gestan y reproducen en el mercado laboral en torno a los trabajos de cuidado. Estos trabajos, distribuidos desigualmente, "no pueden pensarse por fuera de las relaciones de dominación: relaciones asimétricas entre varones y mujeres, pero también entre clases y razas" (Molinier y Legarreta, 2016, p. 6). La profundidad histórica de esas relaciones de dominación permite explicar también las continuidades generacionales dentro de los trabajos de cuidado remunerados.

A su vez, el eje puesto en las largas trayectorias de mujeres peruanas en estos trabajos nos obliga a reflexionar sobre la categoría de tiempo. Cuando nos referimos a largas trayectorias

3 Para un análisis del surgimiento de la perspectiva interseccional y su incidencia en América Latina, véase Busquier (2018), Magliano (2015), Vivero Vigoya (2016). 
estamos dando cuenta de una temporalidad, que en nuestro caso involucra a diferentes generaciones de mujeres dentro de una misma familia que transitan por la misma actividad laboral: los trabajos de cuidado. La propia categoría generación expresa también un orden de tiempo en función de las edades y de las relaciones sociales de los sujetos (Gavazzo, 2014). Si bien esta categoría ha recibido una importante atención académica por parte de distintas disciplinas sociales y humanas, como indican los estudios de Mannheim (1993), Bourdieu (1990), Gavazzo (2014), Zenklusen (2019), entre muchos otros; en este artículo nos interesa especialmente recuperar el sentido temporal de la categoría para poder pensar en clave de larga duración en relación con las continuidades generacionales de mujeres peruanas que se desempeñan en los trabajos de cuidado en Córdoba. El tiempo, sugiere Rufer (2010, p. 13), "es el componente de la memoria". En la reconstrucción de sus trayectorias laborales, entendidas como "la reconstrucción de la vida laboral de los sujetos a lo largo de un período determinado" (Muñiz Terra et al., 2013, p. 61), nuestras interlocutoras pervanas organizan su relato a partir de un orden del tiempo construido desde sus propias experiencias "en un largo proceso de aprendizaje que abarca generaciones" (Elías, 1989, p. 48). Allí, las secuencias que se anudan en torno a los trabajos de cuidados remunerados expresan desde las motivaciones para migrar y las necesidades de subsistencia familiar, hasta las estrategias de reproducción social y las expectativas de las familias peruanas, incluso cuando manifiestan sus deseos de escapar de esos trabajos. Dicho de otro modo, el foco puesto en las largas trayectorias en el sector de los cuidados remunerados por parte de las migrantes peruanas nos permite revisar campos de posibilidades e imposibilidades, constreñimientos sociales en función del género, la clase y la raza, deseos no cumplidos y expectativas truncadas.

En pos de develar estos aspectos, nos basamos en un trabajo de campo cualitativo y longitudinal realizado durante el período 2012-2018, basado principalmente en entrevistas en profundidad con mujeres de origen peruano que residían en la ciudad de Córdoba al momento de la investigación y que se desempeñaban en los trabajos de cuidado remunerados. Ese trabajo de campo se sostuvo especialmente en barrios urbanos relegados y periféricos de la ciudad de Córdoba, donde habitan principalmente nuestras interlocutoras pervanas; y, además, en espacios como el Sindicato de Trabajadoras de Casas de Familia de Córdoba (SINPECAF), que reúne a un conjunto significativo de trabajadoras domésticas pervanas. Las mujeres entrevistadas pertenecían a dos grupos etarios: mujeres adultas entre los 35 y 55 años de edad, y mujeres jóvenes entre los 15 y 25 años de edad. Para el desarrollo de este artículo, recuperamos los testimonios de 6 familias de origen peruano en las cuales tanto madres e hijas se dedicaban a los trabajos de cuidado remunerados (las familias de Mabel y Doris, Lorena y Nancy, Estela y Ashley, Oriana y Gretel, Adriana y Patricia, Marcela y Dana). Además, nos apoyamos en los relatos de 4 mujeres adultas (Teresa, Juana, Daniela y Graciela) y de 4 jóvenes (Carmen, Joselín, Judith y Angie) que se desempeñaban en estos trabajos y a través de las cuales pudimos reconstruir las trayectorias laborales familiares en el sector de los cuidados ${ }^{4}$. A través de las entrevistas en profundidad pudimos reponer las trayectorias migratorias, laborales y los ciclos vitales de las mujeres pervanas -madres e hijas

4 Los nombres de las personas que aparecen en este artículo han sido modificados para preservar su anonimato. 
especialmente- e indagar en las experiencias de incorporación a los trabajos de cuidado 5 . A su vez, como complemento de esta estrategia de recolección de datos, nos valimos de la observación participante, en especial focalizada en reconstruir todo aquello que acontecía durante las entrevistas. La utilización de ambas técnicas fue posible gracias a los marcos de proximidad y confianza que establecimos con las migrantes peruanas quienes nos permitieron acceder al universo más privado de sus vidas.

La permanencia sostenida en el tiempo en el territorio ha sido clave para poder reconstruir las continuidades generacionales en torno a las trayectorias laborales en el sector de los cuidados remunerados. Según Thomson (2007), incorporar la mirada longitudinal contribuye a una exploración más dinámica sobre los cambios y las continuidades de determinados procesos en un contexto temporal y social específico. La propuesta longitudinal cualitativa posibilita captar y explorar en mayor profundidad las situaciones por las que atraviesan las personas, las cuales "pueden alterar sus experiencias y así visibilizar mejor los impactos de unos eventos en diferentes puntos del tiempo" (Farrall, 2006 en Hosnedlová, 2020, p. 118). Por ello, así como las entrevistas en profundidad y la observación participante fueron las técnicas utilizadas para identificar y reconstruir las trayectorias laborales de mujeres peruanas; el trabajo de campo sostenido en el tiempo con estas mujeres facilitó la incorporación de una mirada longitudinal y, en nuestro caso, generacional de esas trayectorias, y así develar las continuidades laborales intrafamiliares dentro de los trabajos de cuidado remunerados.

\section{Las migrantes peruanas y el cuidado remunerado}

La mayoría de las mujeres peruanas adultas que conocimos a lo largo de nuestro trabajo de campo se desempeñaban como trabajadoras del cuidado remunerado, actividad que comenzaron a realizar a partir de la migración hacia Argentina en general y Córdoba en particular. Además, un amplio porcentaje de estas mujeres residía en barrios urbanos relegados y periféricos, espacios hacia donde se reorientó un buen número de migrantes regionales (peruanos, bolivianos y paraguayos principalmente) que llegó a Córdoba (y a otras ciudades argentinas) en las últimas dos décadas. La informalidad que caracteriza a esos espacios -se accede a ellos a través de un proceso de tomas de tierras fiscales- expresa las profundas dificultades que encuentran las poblaciones migrantes que arriban desde la región sudamericana para acceder al mercado formal inmobiliario (Magliano, Perissinotti y Zenklusen, 2014; Magliano y Perissinotti, 2020; Marcos y Mera, 2018; Vaccotti, 2017, entre otras investigaciones) 6 .

5 Si bien en este artículo nos focalizamos en las trayectorias laborales de las mujeres peruanas en Córdoba, vale reconocer que las trayectorias de los varones se ven acotadas también a ciertos sectores del mercado de traba-

jo, al igual que sucede con las mujeres. En particular, se concentran en dos rubros principales: la construcción y la costura. Este dato muestra que los procesos de segregación laboral actúan no solo para las mujeres, sino que también incluyen a aquellos varones sobre los que recaen lógicas de discriminación a partir de la pertenencia de clase, el género, el origen nacional y la adscripción étnico-racial. En cuanto a la distribución de los cuidados no remunerados al interior de las familias, nuestros registros son contundentes: son las mujeres de la familia (madres e hijas) las responsables máximas de estas tareas.

6 La población de origen peruano en la ciudad de Córdoba constituye el colectivo latinoamericano más importante en términos cuantitativos seguida en relevancia por la boliviana (Gómez y Soria, 2017). Según datos del Ins- 
Tanto Teresa como Daniela y Adriana, migrantes peruanas que conocimos cuando comenzamos a realizar el trabajo de campo allá por el 2012, se dedican a los trabajos de cuidado, en especial al trabajo doméstico remunerado por horas. Para las tres, la inserción en este trabajo se abrió a partir de la migración puesto que en Perú realizaban otras actividades laborales: Daniela trabajaba en un organismo público de la Municipalidad Metropolitana de Lima, Adriana cortaba boletos en un colectivo y Teresa se ocupaba del cuidado familiar en su casa. Asimismo, las tres llegaron a Córdoba en el transcurso del período 1995-2005, siendo pioneras de una migración de tipo familiar, dejando en Perú a sus hijos, en general bajo el cuidado de sus propias madres y hermanas, y con miras a una rápida reunificación que el paso del tiempo concretó para Adriana y Teresa7. Juana, Estela, Lorena y Mabel, por su parte, arribaron a Córdoba durante la primera década de este siglo en el marco de proyectos migratorios familiares. Desde los inicios de ese proyecto, se emplean en el sector de los cuidados remunerados. Si bien los trabajos en este sector no formaban parte de los repertorios laborales a los que estas mujeres comúnmente accedían en el contexto pre-migratorio, desde el momento en que comienzan a planificar la migración ya reconocían que posiblemente ese sería su primer trabajo en destino. Es más, muchas de ellas habían conseguido el trabajo como empleadas domésticas y/o cuidadoras incluso antes de migrar. "Yo vine para trabajar en casas de familia" nos comentó Juana en el 2014, "todas sabemos que es fácil conseguir trabajo como doméstica, siempre tienes algún contacto que te consigue trabajo rápido", agregó. En los relatos de nuestras interlocutoras sobre sus primeros recorridos laborales, existe una coincidencia bastante extendida en torno a dos aspectos: por un lado, el necesario tránsito por los trabajos de cuidado una vez que arriban a destino; por el otro, la idea de transitoriedad ligada a estos trabajos al inicio del proyecto migratorio, en especial hasta obtener los documentos y poder encontrar "algo mejor", se va diluyendo conforme pasa el tiempo.

La obtención de un trabajo en el sector de los cuidados, al inicio de la migración, corrió independiente de su situación migratoria. La relativamente fácil convivencia entre los trabajos de cuidado, que se realizan en el ámbito privado del hogar y la irregularidad migratoria, se debe especialmente a la dificultad política de pensar al hogar como un lugar público y, por ende, como un espacio a ser regulado. Como señala Borgeaud-Garciandía (2012, p. 336), el espacio donde se trabaja se construye como un espacio protegido de las intervenciones externas, de la esfera pública y de la mirada del Estado. Esta representación, que puede actuar como una barrera contra la formalidad y la profesionalización del sector (Borgeaud-Garciandía 2012, p. 336), funciona a la vez como un lugar de resguardo frente a los posibles controles en relación con la irregularidad migratoria y laboral. Si bien nuestras interlocutoras tenían la residencia permanente cuando las entrevistamos, al momento de la llegada, la condición de la irregularidad no les impidió trabajar en el sector de los cuidados.

tituto Nacional de Estadísticas y Censos (INDEC), luego de la provincia de Buenos Aires y la Ciudad Autónoma de Buenos Aires, Córdoba es el tercer lugar de destino dentro de la Argentina para la migración peruana. De acuerdo a las estadísticas elaboradas por la Dirección Nacional de Migraciones para el período 201 1-2015, Perú encabezó el listado de radicaciones otorgadas en Córdoba, seguido por Bolivia y Paraguay (DNM, 2016).

7 El proceso de reunificación familiar nunca se concretó para Daniela. Su hija permaneció siempre en Perú al cuidado de su familia. 
Aun cuando todas reconocían el tránsito inexorable por los trabajos de cuidado en sus llegadas a Córdoba, mantenían la expectativa de la transitoriedad. "Me hubiera gustado ejercer mi título acá", reiteró en varias ocasiones Daniela, que es Asistente Social" . "Quería encontrar otro trabajo, pero es muy difícil, no hay otros trabajos para nosotras", apuntó Graciela, otra migrante peruana que conocimos durante el trabajo de campo. "No es que no me guste mi trabajo, pero me gustaría tener otro que me paguen un poco mejor", sentenció Teresa. Y es que los trabajos de cuidado se han distinguido, precisamente, por su precariedad, informalidad y baja remuneración.

A pesar de los intentos por encontrar otros trabajos, no les resultó fácil de lograr. Los impasses temporales fueron cortos $y$, al poco tiempo, tuvieron que retornar a los trabajos de cuidado remunerados como forma de subsistencia familiar. Tal es el caso de Adriana y Juana, quienes abrieron un pequeño kiosco en sus propios domicilios. Sin embargo, y ante la imposibilidad de sostener esos proyectos en el tiempo, los trabajos de cuidado emergían -otra vez- como una opción siempre a mano para estas mujeres. Estos trabajos aparecen entonces como el precario salvavidas en el que las mujeres migrantes configuran su experiencia laboral (Arranz, Carrasco y Massó, 2017, p. 342, énfasis en el original).

Ahora bien, aunque desde sus llegadas a Córdoba las principales inserciones laborales de las mujeres peruanas giraron en torno a los cuidados; sí experimentaron transiciones dentro de este sector: de cama adentro a con retiro por horas; de trabajadoras domésticas a cuidadoras de niños/as y/o ancianos/as; de cualquiera de estas modalidades a cuidadoras comunitarias. En este sentido, las trayectorias expresan una importante circulación al interior de este sector laboral, lo cual se debe a las características principales de este trabajo en relación con su inestabilidad; a los cambios en los proyectos familiares, como por ejemplo cuando se concreta la reunificación familiar; así como a las dificultades de encontrar un empleo por fuera del sector (Magliano, 2017).

Asociado a estas circulaciones, en los últimos años entre las mujeres peruanas (y de los sectores populares en general) se expandió otra modalidad de los trabajos de cuidado remunerado: la comunitaria. El cuidado comunitario remunerado a través de políticas sociales implementadas por los Estados nacionales y provinciales se ha transformado en una alternativa cada vez más requerida por estas mujeres, debido especialmente a que viene a mitigar algunas de las problemáticas más urgentes que moldean sus experiencias de vida en los lugares de destino?. Entre esas problemáticas, se destaca la escasa agencia temporal que poseen, los costos que supone moverse diariamente por la ciudad (teniendo

8 Una cuestión a resaltar en relación con la composición socio-demográfica de la migración peruana, sobre todo aquella que arribó en los años noventa, es (además de la ya mencionada feminización) su calificación. Se trata, en líneas generales, de mujeres con estudios secundarios completos e incluso terciarios y universitarios. En nuestro propio trabajo de campo conocimos maestras, asistentes sociales, secretarias, psicólogas.

9 Nos referimos principalmente al Programa Por mí (Programa de experiencia laboral de jornada corta para mujeres), de alcance provincial; y al Salario Social Complementario, de alcance nacional. 
en cuenta sobre todo que los lugares de trabajo se localizan lejos de los lugares que estas mujeres habitan en las periferias urbanas), y las complicaciones para sostener proyectos familiares en el marco de condiciones laborales precarias (Mallimaci y Magliano, 2019).

Bajo este escenario, las trayectorias migrantes dentro de los trabajos de cuidado revelan el peso de la segmentación interseccional del mercado de trabajo. La configuración social y cultural de las mujeres peruanas como las más aptas para estos trabajos - "las empleadoras llaman al sindicato para pedir migrantes peruanas", nos comentó la representante legal del SINPECAF en el 2014- promueve su encierro en determinados sectores laborales a partir de procesos activos de generización, etnización y racialización de las poblaciones. Procesos que, como veremos en el próximo apartado, alcanzan también a las hijas de estas mujeres. Los escasos márgenes de opción laboral que encuentran las migrantes peruanas en Córdoba (y en Argentina en general) son resistidos a partir de la expectativa de movilidad social ascendente de la mano de la educación de los hijos e hijas, aun cuando en la práctica esto sea difícil de concretar debido a las condiciones de precariedad que afectan las condiciones de vida familiar. En esta tensión entre expectativas y realidad, y al igual que muestra Rosas (2010, p. 156) para el caso del Área Metropolitana de Buenos Aires (AMBA), las jóvenes peruanas tuvieron su primer empleo -incluso a una muy temprana edad- en el sector de los cuidados. Estas largas trayectorias anudan, entonces, (im)posibilidades que revelan el peso de la intersección del género, la clase social y la raza en los recorridos laborales de las familias migrantes pervanas en Córdoba.

\section{De generación en generación: la herencia de los trabajos de cuidado remunerados}

Como señalamos en el apartado anterior, las mujeres pervanas se incorporan al mercado laboral -no bien arriban a la ciudad de Córdoba- desde el sector de los cuidados remunerados y es muy común que sus hijas también comiencen a realizar estos trabajos, primero de manera no remunerada en el contexto barrial, para luego incorporarse de lleno al trabajo remunerado.

Cuando se trata de tareas relacionadas con el sostenimiento de la vida familiar, son las hijas las encargadas de llevarlas a cabo -mientras los/as adultos/as trabajan-, reproduciendo determinados roles anclados en una división de género. En este punto, la cuestión de la edad resulta central, pues es a partir de un cierto momento que las personas adultas comienzan a delegar tareas de cuidado en sus hijas: cocinar, limpiar, realizar compras, cuidar de sus hermanos/as. Las hijas se convierten en las verdaderas responsables de la reproducción doméstica de la familia, permitiendo por este medio que sus madres asuman el rol de proveedoras a través de los trabajos de cuidado ${ }^{10}$. El involucramiento en estas tareas expresa las diferentes responsabilidades que asumen las hijas-además de asistir a

10 Con esto no queremos decir que las mujeres sean las únicas proveedoras, sino que comparten ese rol con los adultos varones. 
la escuela- y los esfuerzos que realizan: "por la mañana le cocino a mis hermanos y a la tarde cuando vuelvo de la escuela aprovecho y estudio, porque mi mamá ya está en casa", sostuvo Nancy. La mamá de Nancy, Lorena, trabaja cuidando a una anciana. "Yo cuidaba de mis hermanos mientras mamá trabajaba cuidando niños en la guardería. Un día vino la vecina y también me preguntó si podía cuidar de su hijo por unos pesos", comentó Dana en una oportunidad. "Mi mamá me enseñó a cocinar porque, cuando ella consiguió el trabajo, tuve que cocinarle a mis hermanos y también a los vecinitos", nos contó Gretel. Su madre, Oriana, migrante peruana que llegó a Córdoba en el transcurso de la primera década del siglo XXI, trabaja por horas y para distintos empleadores en el centro de la ciudad de Córdoba limpiando departamentos. Relatos como estos se multiplican entre las jóvenes peruanas dando cuenta de la persistencia de una división sexual del trabajo en torno a los cuidados.

A su vez, en esos relatos aparecen dos dimensiones que merecen ser atendidas. En primer lugar, las jóvenes entrevistadas señalaron que a partir de cuidar de sus hermanos/as más pequeños y de realizar las tareas del hogar adquirieron un saber hacer, que en ocasiones derivó en el cuidado remunerado de otros niños y niñas del barrio. Las hijas lentamente y desde temprana edad aprenden de esos trabajos. En segundo lugar, a partir de la migración, las trayectorias de sus madres están marcadas por el cuidado remunerado en casas particulares o en los merenderos, comedores y guarderías infantiles del barrio. Así, la participación de las jóvenes cuidando de hermanos/as menores o sobrinos/as funciona como una estrategia de reproducción familiar en contextos de migración y como el puntapié para que emerjan redes de ayuda mutua que operan a nivel territorial para el cuidado. Tradicionalmente, y como bien señala Faur (2012), la ayuda recíproca entre familiares y vecinos/as ha sido una estrategia frecuente para paliar necesidades de distinta índole. Dentro de los sectores empobrecidos, el contar con el cuidado de hermanas, tías y sobrinas resulta indispensable para la salida laboral de muchas de estas mujeres. Si bien las tareas que realizan las hijas podrían pensarse solo en términos de redes de ayuda mutua, lo cierto es que las jóvenes reconocieron recibir una remuneración -aunque modesta- como contraprestación por esa tarea: "ahora en verano que estamos de vacaciones mi mamá [migrante pervana que se dedica al trabajo de limpieza por horas en casas particulares] trabaja a full y aprovecha a juntar plata. Y yo aprovecho y cuido de dos vecinitos también para hacer algo de platita", reconoció Carmen. De esta manera, estas experiencias vinculadas al cuidado se tornan centrales en las trayectorias de las jóvenes y comienzan a adquirir el sentido de un trabajo remunerado.

Otra dimensión que aparece en las trayectorias laborales de algunas jóvenes es la articulación con determinadas políticas sociales. Al igual que las madres, las hijas acceden a programas de trabajo, como el Programa Primer Paso (PPP) ${ }^{11}$, destinados exclusivamente a los y las jóvenes. Las beneficiarias a cambio de una retribución económica, realizan una contraprestación en algunas de las instituciones que forman parte del programa de acuer-

11 El Programa Primer Paso (PPP) contempla a jóvenes residentes en Córdoba entre los 16 y 25 años y apunta a concretar su primera experiencia laboral. 
do a los cupos y a los intereses individuales es pos de adquirir experiencia laboral. En nuestro caso, varias de las jóvenes reconocieron que por medio de algunos programas se sumaron a trabajar en los comedores, merenderos y guarderías barriales como parte de esa contraprestación, realizando tareas de cuidado comunitario remunerado. Allí, con menos horas que las mujeres adultas, desarrollan actividades de limpieza, aportan en la cocina y acompañan con el cuidado de niños y niñas.

Al igual que sucede con sus madres, estos primeros trabajos, que usualmente realizan en paralelo con su educación secundaria, son configurados como transitorios. Es decir, les sirve a las jóvenes para contar con sus propios ahorros y contribuir a la economía familiar. En sus relatos, las jóvenes enfatizan que no quieren trabajar cuidando niños o limpiando casas. "Por ahora cuido al bebé de un vecino y voy a la escuela, pero quiero estudiar para ser psicóloga", narraba Doris, una joven peruana, hija de Mabel, una trabajadora doméstica; "a mí me gustaría ser abogada, el trabajo en la guardería cuidando niños me cansa, me hago unos pesitos, pero no me gusta", señalaba Angie.

Sin embargo, no siempre esa transitoriedad se concreta en la práctica: las dificultades económicas para sostener los proyectos universitarios, las maternidades tempranas y las propias necesidades de subsistencia familiar truncan muchas veces los deseos y expectativas de las jóvenes peruanas. En este marco, los trabajos de cuidado remunerados adquieren un carácter de más largo plazo que no poseían al inicio ni para ellas ni para el resto de su familia.

Como estos testimonios lo indican, muchas de estas jóvenes, una vez finalizada la escuela secundaria, se proponían continuar con estudios universitarios y terciarios. Si bien la educación de las hijas -y también de los hijos- resulta central para las familias pervanas en su conjunto y forma parte del proyecto migratorio familiar (Zenklusen, 2020), en la práctica sostener trayectorias educativas en el nivel superior no es una tarea sencilla. La mayoría de las jóvenes que accedieron a los estudios superiores, lo hacía en simultáneo con su incorporación en trabajos de cuidado remunerados por fuera de los barrios. Esto les permitía un mayor ingreso - a diferencia del que obtenían cuidando vecinos/as o por medio de los programas sociales en trabajo comunitario- para solventar parte de su carrera universitaria o terciaria y continuar aportando a la economía familiar. "Empecé a estudiar enfermería en Villa el Libertador cuando terminé la escuela, y de ahí conseguí algunas changas en geriátricos por las noches", indicó Ashley, hija de Estela, una migrante peruana que se dedica al trabajo de doméstico en casas particulares. En ocasiones, y como señala Mallimaci (2018), para las mujeres migrantes estudiar carreras vinculadas al cuidado se presenta como una salida, como una posibilidad de alcanzar una movilidad ascendente por medio de la profesionalización de aquello que ya vienen realizando. No obstante, algunas de ellas debieron abandonar o postergar sus estudios para priorizar el sustento económico o, en algunos casos, la llegada de un hijo/a. "Cuando terminé el secundario comencé a estudiar cuidados gerontológicos, pero quedé embarazada y, al poquito tiempo que nació Gian, empecé a trabajar en la casa de una señora, limpiaba por horas", mencionó Joselin. 
Otro grupo de jóvenes finalizado el secundario buscó incorporarse a otros sectores del mercado de trabajo -costura, comercio, salones infantiles, supermercados, ventas de comidas. A pesar de ello, los trabajos en cuidado remunerados, ya sea por la red de personas que facilitaba su ingreso (madres, tías y vecinas que ya trabajaban de eso), porque era menos intermitente o por los imaginarios que persisten en torno a las trabajadoras peruanas como las más aptas para estos trabajos, se convierten en la mejor opción para ellas. "Una vecina me dijo que buscaban muchachas y ahí fui. Trabajo en una empresa de limpieza, limpiando un lugar de atención a enfermos", explicó Judith, una joven peruana que comenzó a trabajar finalizado el secundario mientras su mamá se dedicaba al cuidado de adultos mayores.

En las trayectorias laborales de las hijas existen barreras visibles e invisibles que moldean esas búsquedas. En todos los relatos el punto de enlace era que el trabajo de cuidado remunerado se presentaba como una posibilidad rápida y rentada de una práctica que incorporaron y aprendieron a partir de la propia experiencia en sus casas y en el barrio. Al igual que Zibecchi (2014), entendemos a la familia como el ámbito donde se heredan relaciones y prácticas vinculadas al cuidado del otro. Y, generalmente, son los vínculos personales establecidos con las mujeres de las familias y con vecinas también peruanas los que permiten la circulación de información, contactos y recomendaciones para la incorporación en este sector laboral. Aun cuando estas jóvenes despliegan diferentes estrategias -como el ingreso a la universidad o la búsqueda de otros trabajos- pareciera que sobre ciertas trayectorias laborales recae la herencia generacional de las madres. Estas continuidades dan cuenta de las dificultades con las que se topan los colectivos migrantes (y no solo ellos) para moverse hacia otros empleos -presumiblemente- de mayor calificación, con mejores salarios y/o condiciones laborales.

\section{Conclusiones}

Este artículo reconstruyó las largas trayectorias de cuidado remunerado de las mujeres peruanas en la ciudad de Córdoba en Argentina. Con base en un estudio cualitativo y longitudinal, repuso la relevancia de la dimensión generacional para analizar los largos vínculos temporales que estas mujeres establecen con los trabajos de cuidado. En tal sentido, mostramos la centralidad del cuidado en las experiencias de las migrantes pervanas que arribaron a Córdoba desde la década del noventa en adelante, convirtiéndose en muchos casos en la primera -y única- inserción laboral en el contexto de llegada. Al igual que sucede en el ámbito global, la mayoría de las mujeres migrantes (y también los varones) se ha orientado hacia aquellas actividades del mercado peor remuneradas y con mayores niveles de inestabilidad laboral, revelando cómo la etnicidad, la raza, el género y el origen nacional funcionan como marcadores de posibilidades y de posiciones en la distribución de recursos en los países de destino (Pedreño Cánovas, 2005). Los trabajos de cuidado, en este marco, se convierten en marcadores de (im)posibilidades para estas mujeres y también

para sus hijas, expresando el modo en que la generización, etnización y racialización del mercado de trabajo derivan en la constitución de nichos laborales con alta participación de 
población migrante. Nichos laborales que, a su vez, se retroalimentan generacionalmente. Esa retroalimentación descansa especialmente en redes familiares y vecinales que facilitan la obtención de determinados trabajos, como sucede con los de cuidado.

En el artículo, justamente, apuntamos a visibilizar las continuidades generacionales en torno a esos trabajos. Así pues, indicamos que el cuidado sigue siendo para las jóvenes peruanas el trabajo de más fácil acceso. Pese a las expectativas propias y familiares vinculadas con la posibilidad de acceso a los estudios superiores y con la oportunidad de conseguir otros trabajos, diferentes a aquellos de sus madres y familiares cercanos; para las jóvenes peruanas no es sencillo escapar de los cuidados remunerados como opción laboral. Éstos, como vimos, comienzan siendo no remunerados y configurados como una ayuda familiar, para lvego ir adquiriendo un carácter (sub)remunerado e impulsados, en muchos casos, por las mismas políticas sociales. Estas continuidades generacionales en sectores del mercado de trabajo que se distinguen por su precariedad e inestabilidad nos invitan a pensar críticamente sobre los alcances de la segregación laboral entre las poblaciones migrantes. Trabajos configurados como provisorios -primero por las mujeres peruanas adultas pioneras de la migración y luego por sus hijas, sobre quienes se depositan expectativas de movilidad social ascendente- pero que, en la práctica y a partir del peso de los constreñimientos de género, clase y raza, se vuelven permanentes. Es esa permanencia, o la imposibilidad de la transitoriedad, sostenida generacionalmente lo que nutre la larga duración.

\section{Bibliografía}

Arranz, J., Carrasco, C. y Massó, M. (2017) La movilidad laboral de las mujeres inmigrantes en España (2007-2013). Revista Española de Sociología, 26 (3), 329-344. doi: https://doi.org/10.22325/fes/res.2017.26

Arruzza, C., Bhattacharya, T. y Fraser, N. (2019). Manifiesto de un feminismo para el 99\%. Barcelona, España: Herder.

Baeza, B. (2013). Trabajadores de la construcción: bolivianos y paraguayos en Comodoro Rivadavia. Identificaciones, diferenciaciones y conflictos. En $G$. Karasik (coord.). Migraciones internacionales. Reflexiones y estudios sobre la movilidad territorial contemporánea (pp. 361-382). Buenos Aires, Argentina: CICCUS.

Borgeaud-Garciandía, N. (2018). Introducción. En N. Borgeaud-Garciandía (comp.). El trabajo de cuidado (pp. 13-29). Buenos Aires, Argentina: Fundación Medifé Edita.

- (2017). Puertas adentro. Trabajo de cuidado domiciliario a adultos mayores y migración en la Ciudad de Buenos Aires. Buenos Aires, Argentina: Teseo. . (2012). La cuidadora domiciliaria de ancianos: de poca visibilidad de su desempeño laboral. Trabajo y sociedad, (19), 321-344. Recuperado de https:// www.unse.edu.ar/trabajoysociedad/19\%20BORGEAUD-GARCIANDIA\%2Oinvisibilidad\%20laboral\%20cuidadoras\%20de\%20ancianos.pdf

Bourdieu, P. (1990). Sociología y cultura. Ciudad de México, México: Grijalbo.

Busquier, L. (2018). ¿Interseccionalidad en América Latina y el Caribe? La experiencia de la Red de Mujeres Afrolatinoamericanas, Afrocaribeñas y de la Diáspora desde 1992 hasta la actualidad. Con X, (4), 1-21. doi: https://doi. org/10.24215/24690333e023

Catarino, C. y Oso, L. (2000). La inmigración femenina en Madrid y Lisboa: hacia una etnización del servicio doméstico y de las empresas de limpieza. Papers, (60), 183-207. doi: http://dx.doi.org/10.5565/rev/papers/v60n0.1038

Castles, S. (2013). Migración, trabajo y derechos precarios: perspectiva histórica 
y actual. Migración y Desarrollo, 11 (20), 8-42. Recuperado de http://www. scielo.org.mx/scielo.php?script=sci_arttext\&pid=\$1870-75992013000100002

Cerrutti, M. (2005). La migración peruana a la Ciudad de Buenos Aires: su evolución y características. Población de Buenos Aires, Dirección General de Estadísticas y Censos, 2 (2), 7-28. Recuperado de https://www.researchgate.net/ publication/242620392_La_migracion_pervana_a_la_Ciudad_de_Buenos_Aires_su_evolucion_y_caracteristicas

Courtis, C. y Pacecca, M. I. (2010). Género y trayectoria migratoria: mujeres migrantes y trabajo doméstico en el Área Metropolitana de Buenos Aires. Papeles de Población, 16 (63), 155-185. Recuperado de http://www.scielo.org.mx/ scielo.php?script=sci_arttext\&pid=S1 405-74252010000100006

DNM. (2016). Panorama Radicaciones 2011-2015. Buenos Aires: Ministerio del Interior, Obras Públicas y Vivienda. Recuperado de http://www.migraciones.gov. ar/pdf/estadisticas/radicaciones_2011-2015.pdf

Dutra, D. y Matos, R. (2016). Trabajo doméstico y migración en Brasil. Contexto histórico-legal y mercado de trabajo. En M. J. Magliano, M. V. Perissinotti y D. Zenklusen (comps). Los nudos ciegos de la desigualdad. Diálogos entre migraciones y cuidados (pp. 51-82). Buenos Aires, Argentina: CONICET.

Elías, N. (1989). Sobre el tiempo. Madrid, España: Fondo de Cultura Económica.

Falcón, M. del C. y Bologna, E. (2013). Migrantes antiguos y recientes: una perspectiva comparada de la migración peruana a Córdoba, Argentina. Migraciones Internacionales, 7 (1), 235-266. doi: http://dx.doi.org/10.17428/rmi. v6i24.715

Faur, E. (2012). El cuidado infantil desde las perspectivas de las mujeres-madres. Un estudio en dos barrios populares del Área Metropolitana de Buenos Aires. En V. Esquivel, E. Faur y E. Jelin (eds). Las lógicas del cuidado infantil. Entre las familias, el Estado y el mercado, (pp. 107-164). Buenos Aires, Argentina: IDES.

Fulladosa-Leal, K. (2013). Mujeres en movimiento. Ampliando los márgenes de participación social y política en la acción colectiva como trabajadoras del hogar y el cuidado (Tesis Doctoral). Barcelona, España: Universidad Autónoma de Barcelona.

Gavazzo, N. (2014). La generación de los hijos: identificaciones y participación de los descendientes de bolivianos y paraguayos en Buenos Aires. Sociedad y equidad, (6), 58-87. doi: 10.5354/0718-9990.2014.27263

Gómez, P. y Soria, D. (2017). Cambios y continuidades en la incorporación espacial de los migrantes pervanos en la ciudad de Córdoba, Argentina. REMHU, 25 (50), 133-156. doi: https://doi.org/10.1590/1980-85852503880005009

Gutiérrez-Rodríguez, E. (2010). Migration, Domestic Work and Affect: A Decolonial Approach on Value and the Feminization of Labor. New York, Estados Unidos: Routledge.

Herrera, G. (2016). Trabajo doméstico, cuidados y familias transnacionales en América Latina: reflexiones sobre un campo en construcción. Amérique Latine Histoire et Mémoire. Les Cahiers ALHIM 31. Recuperado de http://alhim. revues.org/5430

Hochschild, A. (2000). Global care chains and emotional surplus valuate. En A. Giddens y W. Hutton (eds.). On the Edge: Globalization and the New Millennium, (pp. 130-146). London, UK: Sage Publishers.

Hosnedlová, R. (2020). El proceso intencional/decisorio migratorio desde una aproximación cualitativa longitudinal. Empiria. Revista de Metodología de Ciencias Sociales, (46), 115-145. doi: https://doi.org/10.5944/empiria.46.2020.26969

Magliano, M. J. (2017). Las trabajadoras invisibles: experiencias laborales de mujeres migrantes en Argentina. Revista Latinoamericana de Antropología del Trabajo, 1 (1), 1-23. Recuperado de http://www.ceil-conicet.gov.ar/ojs/index. $\mathrm{php} /$ lat/article/view/228

(2015). Interseccionalidad y migraciones: potencialidades y desafíos. Revista Estudos Feministas, 23 (3), 691-712. doi: https://doi.org/10.1590/0104-026 X2015v23n3p691

Magliano, M. J. y Mallimaci, A. I. (2018). Segregación laboral. Revista Temas de Antropología y Migración, (10), 13-19. Recuperado de http://www.migrantropologia.com.ar/images/stories/PDF/Revista10/partel.pdf

Magliano, M. J. y Perissinotti, M. V. (2020). La periferia autoconstruida: migraciones, informalidad y segregación urbana en Argentina. Eure. Revista Latinoa- 
mericana de Estudios Urbanos Regionales, 46 (138), 5-23. Recuperado de file:///C:/Users/pc/Downloads/3147-20944-1-PB.pdf

Magliano, M.J., Perissinotti, V. y Zenklusen, D. (2016). Los nudos ciegos de la desigualdad. Diálogos entre migraciones y cuidados. Buenos Aires, Argentina: CONICET.

. (2014). Estrategias en torno a las formas de apropiación y organización del espacio en un 'barrio de migrantes' de la ciudad de Córdoba, Argentina. Revista Estudios Demográficos y Urbanos, 29 (3), 513-539. doi: http://dx.doi. org/10.24201/edu.v29i3.1470

Maguid, A. (2011). Migrantes sudamericanos y mercado de trabajo. En La inmigración laboral de sudamericanos en Argentina (pp. 109-130). Buenos Aires, Argentina: OIT/Ministerio de Trabajo, Empleo y Seguridad Social.

Mallimaci, A. I. y Magliano, M. J. (agosto de 2019). La espera y las migraciones. En V. Trpin, (Directora), VI Seminario de migraciones internacionales contemporánea: enfoques, perspectivas y metodologías para la investigación, Centro de Investigaciones y Estudios sobre Cultura y Sociedad (CIECS / CONICET y UNC), Córdoba, Argentina.

Mallimaci, A. I. (2018). Circulaciones laborales de mujeres migrantes en Buenos Aires: de empleadas domésticas a enfermeras. Cadernos Pagu, (54), 1-33. doi: https://doi.org/10.1590/18094449201800540012.

Manheim, K. (1993) [1928]. El problema de las Generaciones. Revista española de Investigaciones Sociológicas, (62), 193-242. Recuperado de https://dialnet. unirioja.es/servlet/articulo? codigo $=766796$

Marcos, M. y Mera, G. (2018). Migración, vivienda y desigualdades urbanas: condiciones socio-habitacionales de los migrantes regionales en Buenos Aires. Revista INVI, 33 (92), 53-86. doi: http://dx.doi.org/10.4067/S0718-83582018000100053

Martín Palomo, M. T. (2016). Cuidado, vulnerabilidad e interdependencia. Nuevos retos políticos. Madrid, España: Centro de Estudios Políticos y Constitucionales.

Mezzadra, S. y Neilson, B. (2016). La frontera como método. O la multiplicación del trabajo. Buenos Aires, Argentina: Tinta Limón.

Molinier, P. (2018). El cuidado puesto a prueba por el trabajo. Vulnerabilidades cruzadasy saber-hacer discretos. En N. Borgeaud-Garciandía (comp.). El trabajo de cuidado (pp. 187-201). Buenos Aires, Argentina: Fundación Medifé Edita.

Molinier, P. y Legarreta, M. (2016). Subjetividad y materialidad del cuidado: ética, trabajo y proyecto político. Papeles del CEIC, (1), 1-14. doi: http://dx.doi. org/10.1387/pceic.16084

Muñiz Terra, L., Roberti, E., Deleo, C. y Hacisic, C. (2013). Trayectorias laborales en Argentina: una revisión de estudios cualitativos sobre mujeres y jóvenes. Revista Lavboratorio, 25 (14), 57-79. Recuperado de https://publicaciones.sociales.uba.ar/index.php/lavboratorio/article/view/119

Parella, S. (2007). Los vínculos afectivos y de cuidado en las familias transnacionales Migrantes ecuatorianos y peruanos en España. Migraciones Internacionales, 4 (2), 151-188. Recuperado de http://www.scielo.org.mx/pdf/migra/v4n2/ $\checkmark 4 n 2 a 6 . p d f$

Parreñas, R. (2001). Servants of globalization. Women, migration and domestic work. Standford, Estados Unidos: Standford University Press.

Pedone, C. (2014). Rupturas y continuidades de los roles de género en contextos migratorios transnacionales. Relatos sobre sexualidad y salud reproductiva de los hijos e hijas de la inmigración ecuatoriana en Cataluña. Papeles del CEIC. (2), 1-38. Recuperado de https://www.ehu.eus/ojs/index.php/papelesCEIC/ issue/view/1232

Pedreño Cánovas, A. (2005). Sociedades etnofragmentadas. En A. Pedreño Cánovas y M. Hernández Pedreño (coords.), La condición inmigrante. Exploraciones e investigaciones desde la Región de Murcia (pp. 75-106). Murcia, España: Universidad de Murcia.

Pérez Orozco, A. (2014). Subversión feminista de la economía. Aportes para un debate sobre el conflicto capital-vida. Madrid, España: Traficantes de Sueños.

Rodríguez Enríquez, C. y Sanchís, N. (2011). El papel de las migrantes paraguayas en la provisión de cuidados en Argentina. Santo Domingo, República Dominicana: ONU Mujeres. 
Rosas, C. y Gil Araujo, S. (2019). La migración peruana en la República Argentina. Perfil sociodemográfico, acceso a derechos y acción colectiva. Buenos Aires, Argentina: OIM/IIGG.

Rosas, C. (2010). Implicaciones mutuas entre el género y la migración. Mujeres y varones pervanos arribados a Buenos Aires entre 1990 y 2003. Buenos Aires, Argentina: Eudeba.

Rufer, M. (2010). La temporalidad como política: nación, formas de pasado y perspectivas poscoloniales. Memoria y Sociedad, 14 (28): 11-31. Recuperado de https://revistas.javeriana.edu.co/index.php/memoysociedad/article/ view/8247

Segato, R. (2007). La nación y sus otros. Buenos Aires, Argentina: Prometeo.

Stolcke, V. (2000). ¿Es el sexo para el género como la raza para la etnicidad? Cuadernos para el Debate, (6), 5-32. Recuperado de https://www.redalyc.org/articulo. oa?id $=26701403$

Thomson, R. (2007). The qualitative longitudinal case history: Practical, methodological and ethical reflections. Social Policy and Society, 6 (4), 571-582. Doi: https://doi.org/10.1017/S1474746407003909

Trpin, V. y Pizarro, C. (2017). Movilidad territorial, circuitos laborales y desigualdades en producciones agrarias de argentina: abordajes interdisciplinares y debates conceptuales. REMHU, Rev. Interdiscip. Mobil. Hum., Brasilia, 25 (49), 35-58. doi: http://dx.doi. org/10.1590/1980-85852503880004903.

Vaccotti, L. (2017). Migraciones e informalidad urbana. Dinámicas contemporáneas de la exclusión y la inclusión en Buenos Aires. Eure. Revista Latinoamericana de Estudios Urbanos Regionales, 43 (129), 49-70. doi: http://dx.doi. org/10.4067/S0250-71612017000200003.

Vega, C. y Gutiérrez-Rodríguez, E. (2014). Nuevas aproximaciones a la organización social del cuidado. Debates latinoamericanos. Íconos. Revista de Ciencias Sociales, (50), 9-26. Doi: 10.17141/iconos.50.2014.1425

Vivero Vigoya, M. (2016). La interseccionalidad: una aproximación situada a la dominación. Debate Feminista, (52), 1-17. doi: https://doi.org/10.1016/j. df.2016.09.005

Zenklusen, D. (2019). Hijos/as de la migración. Rupturas y continuidades en las relaciones de género y generacionales en familias migrantes de origen peruano en la ciudad de Córdoba (Tesis de doctorado). Facultad de Filosofía y Letras, Universidad de Buenos Aires, Buenos Aires, Argentina.

Zenklusen, D. (2020). "Quiero seguir estudiando para ser alguien": análisis de trayectorias educativas de jóvenes peruanos en Argentina. Revista Latinoamericana de Ciencias Sociales, Niñez y Juventud (RLCSNJ), 18 (2), 1-27. doi: http:// dx.doi.org/10.11600/1692715x.18203

Zibecchi, C. (2014). Mujeres cuidadoras en contextos de pobreza: el caso de los Programas de Transferencias Condicionados en Argentina. Revista Estudios Feministas, 22 (1), 91-1 13. Doi: https://doi.org/10.1590/S0104-026X2014000100006. 